\title{
Impact of high index buffer layer on the improvement of the MMI sensor sensitivity
}

\author{
Artur Szewczuk, Marek Błahut \\ Department of Optoelectronics, Silesian University of Technology, Akademicka 2A, 44-100 Gliwice
}

Received November 12, 2014; accepted December 29, 2014; published December 31, 2014

\begin{abstract}
The results of investigations are presented, of the impact of high index buffer layer on the improvement of the multimode interference (MMI) sensor sensitivity. The basis of the presented research is a gradient index MMI structure with a multimode section length relating to the length of an input field image formation. The multimode section is covered with a material which changes its optical parameters (refractive index and extinction coefficient) when exposed to appropriate gaseous environments. The coating parameters have an effect on light propagation conditions in a structure, which can be seen as an output signal variation. In this configuration a thin buffer layer of high refractive index is placed between the sensing layer and the gradient core. We present the results showing how these buffer layers lead to a sensitivity increase in the sensor based on the MMI structures. The performed analyses are based on numerical BPM calculations.
\end{abstract}

At present, strong emphasis is placed on environmental monitoring. This affects the rapid development of sensor technology. Among the many developed sensor technologies, waveguide sensors used in integrated optics (IO) technology have growing importance. These sensors show many advantages. First of all, they have a small size, are resistant to electromagnetic interference, and are characterized by a very fast response [1-2]. In addition, a sensor signal can be transmitted by optical fibers, which in many cases significantly simplifies the structures and allows applying a network of sensors that can monitor various physical parameters from a larger area.

There are many configurations of such sensors. Especially popular are configurations of sensors based on IO interferometers [3], surface plasmon resonance [4] and evanescent field spectroscopy in strip waveguides [5].

Moreover, very prospective is the possibility of developing a new class of optical sensors - those of multimode interference. These sensors were examined in [6-9] for gradient index structures made by $\mathrm{K}^{+}-\mathrm{Na}^{+}$ technology. In paper [6] the influence of the geometry and size of multimode interference (MMI) structures on the operation characteristics of the proposed sensor was analyzed. In [7-8] MMI structures covered with a thin metal oxides layer were investigated. In such configurations, the output signal depends on the extinction of coefficient changes. In [9] the ammonia sensor based on a thin bromocresol purple sensing layer has been presented. The tested sensor structure was characterized by high sensitivity at low concentrations of ammonia.
In this paper we discuss the MMI sensor configuration with a thin buffer layer of high refractive index, which can be a way of improving MMI sensor sensitivity.

Multimode interference structures are composed of three groups of waveguides: single mode input and output waveguides and a multimode waveguide placed between them. The simplest MMI waveguide sensor configuration based on the structure consists of a single input and output waveguide.

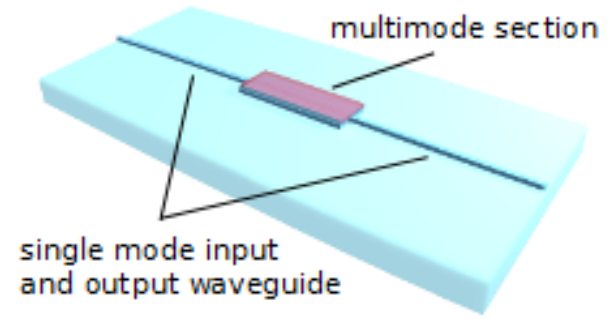

Fig.1. Configuration of the sensor structure.

In the case of symmetrical interference configuration in question, single mode waveguides are placed centrally relative to the multimode section (Fig. 1). The length of the multimode section is selected in order to obtain, at the output, a single image of the input field. The MMI section is covered with a dielectric layer which changes its refractive index with changes of concentration of the measured analyte.

This configuration was analyzed in many scientific studies, both by the authors of this publication and by other research groups [10]. The modifications of the refractive index of the sensing layer influence the mode properties of a multimode waveguide, and thus affect position changes of sn input field image, as shown in Fig. 2. It can be seen that for a higher value of the refractive index of the cladding, the propagation length increases for the input field image formation. 


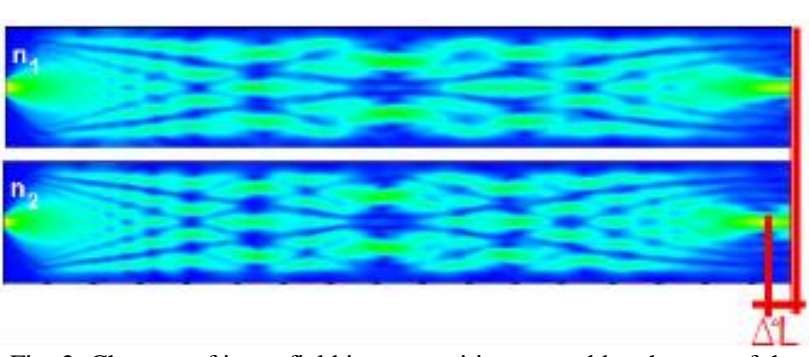

Fig. 2. Changes of input field image position caused by changes of the cladding refractive index for the refractive indices $n_{1}>n_{2}$.

The considered structures were based on the gradient waveguides made in the $\mathrm{K}^{+}-\mathrm{Na}^{+}$ion exchange process [11]. The process and geometry parameters have been determined in order to obtain single mode input and output waveguides.

Crucial for the image position of an input field is the difference between propagation mode constants [7], related to their effective refractive indices $\mathrm{N}_{\text {eff. }}$. The values of the effective refractive indices are within the range between the refractive index of the substrate and the maximum refractive index of the gradient core. For gradient index waveguides made by $\mathrm{K}^{+}-\mathrm{Na}^{+}$ion exchange, it amounts to 0.01. Therefore, for such structures, even large changes of the refractive index of the cover will not affect the distinct change of $\mathrm{N}_{\text {eff }}$.

$\mathrm{Ab}$ increase in sensitivity can be achieved by applying a thin layer of a high refractive index located between the sensing layer and the gradient core (Fig. 3). The buffer layer must be sufficiently thin so that the structure is still single mode for the direction perpendicular to the substrate.

The investigated MMI sections are produced in the $\mathrm{K}^{+}$ $\mathrm{Na}^{+}$ion exchange process in a time of $0.75 \mathrm{~h}$ and temperature of $400^{\circ} \mathrm{C}$ in borosilicate glass. The distribution profile of the refractive index of the MMI section obtained in the diffusion process is calculated numerically from the nonlinear diffusion equation:

$$
\frac{\partial C_{K}}{\partial t}=\nabla\left[\frac{D_{K}}{1-(1-m) \cdot C_{K}} \cdot \nabla C_{K}\right],
$$

where $C_{\mathrm{K}}$ is the dopant $\mathrm{K}^{+}$ions concentration, proportional to the refractive index change. The material parameters of the technological process: the self-diffusion coefficient of $\mathrm{K}^{+}$ions $D_{\mathrm{K}}$, the mobility ratio $m$ of the ions $\mathrm{K}^{+}$and $\mathrm{Na}^{+}$, and the maximum of the refractive index change, were determined by the measurements of respective planar index profiles using an IWKB method [12]. The calculations of mode field distribution, performed by an effective gradient index method show that the presence of a buffer layer changes the field distribution of modes, moving them towards the cover.

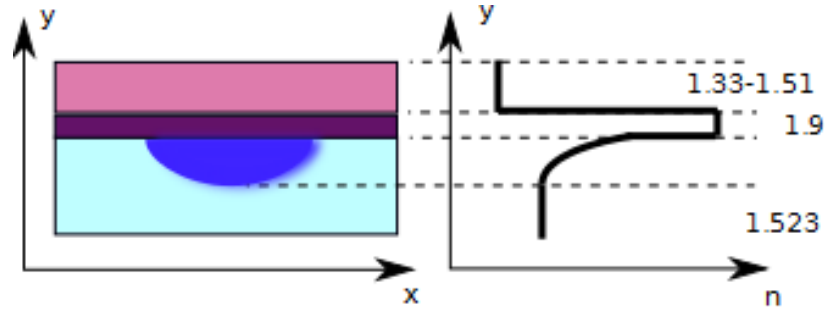

Fig. 3. Refractive index configuration of the MMI sensor.

The effect is shown in Fig. 4. The boundary between the glass and the coating is indicated in the graph by the dotted line. This configuration results in a larger shift of the input field image position associated with the refractive index changes of the active cover.

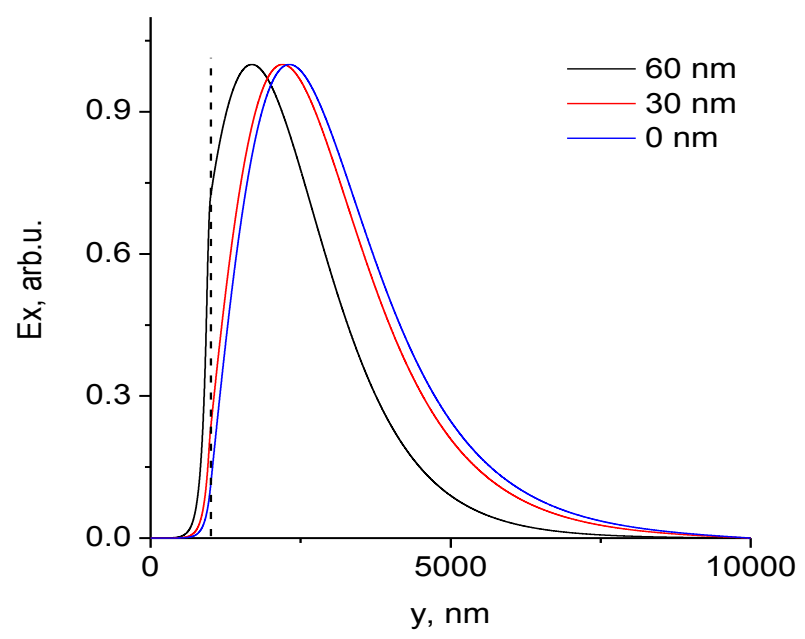

Fig. 4. Mode function distribution in the MMI section in the direction perpendicular to the substrate; calculations performed for various thicknesses of the buffer layer.

The presented wave functions were calculated for a multimode section $45 \mu \mathrm{m}$ wide, without a buffer layer and with a buffer layer with the refractive index of 1.9, 30 and $60 \mathrm{~nm}$ thick.

Theoretical analysis was carried out for MMI structures coated with thin layers of the 1.9 refractive index. The refractive index of the active cladding layer above the buffer layer varied in the range of 1.33-1.51. The buffer and sensor layer covered only the multimode section area. The refractive index of the input and output waveguides cladding was equal to one.

The number of modes excited in the direction perpendicular to the substrate depends on the refractive indices of the coating and the buffer layer. Figure 5 presents the propagation length $L$ for the single input field image formation, for TE modes as a function of the 
refractive index of the cladding, numerically calculated on the basis of equation [13] :

$$
L=p\left(3 L_{\pi}\right) \quad p=0,1,2, \ldots,
$$

where $\mathrm{L}_{\pi}$ is the beat length of the lowest order modes.

Calculations were performed for the MMI section covered with buffer layers of different thicknesses. The range of refractive index changes of the cover responds to a single mode operation of the structure for the direction perpendicular to the substrate. One can see that the an increase in the thickness of the buffer layer brings about more dynamic changes in image position of an input field associated with refractive index changes of the environment.

The structure is a single mode for the direction perpendicular to the substrate, in a whole range of changes of the refractive index of the cladding for the buffer layers with a refractive index of 1.9 and a thickness of $15 \mathrm{~nm}$.

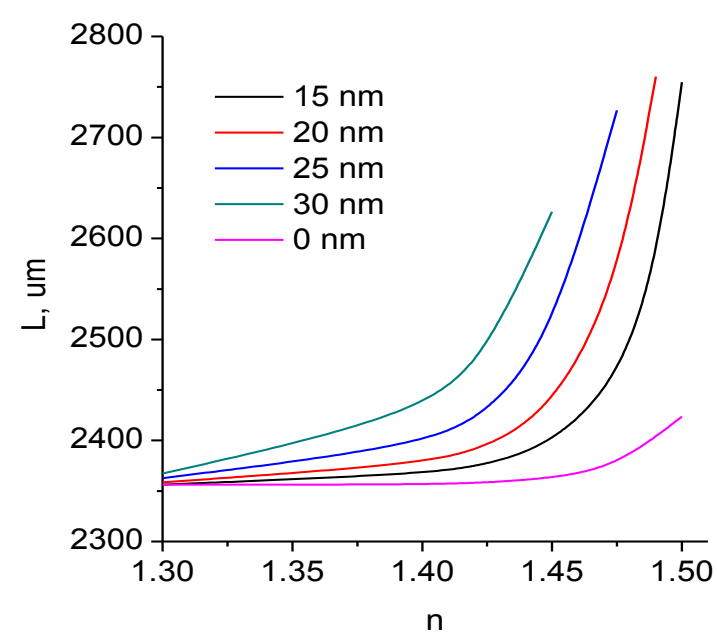

Fig. 5. Input field image position as a function of the refractive index of the cover for different buffer layer thickness.

There was no significant image shift of the input field for the structure without a buffer layer for all the refractive index changes of the cover. Better results were obtained for the configuration with a buffer layer. Numerically calculated by the BPM method, the dependence of the output signal intensity as a function of refractive index of the cladding, for the structures with buffer layers of various thicknesses is shown in Fig. 6. It can be seen that the use of buffer layers significantly increases the sensitivity of analyzed configurations.

The structure without a buffer layer allows to determine refractive index changes of the cladding only for the values close to the refractive index of the substrate glass.
However, the output signal changes for the structure with a $30 \mathrm{~nm}$ buffer layer allow to specify the cladding parameters in the whole analyzed range of a refractive index.

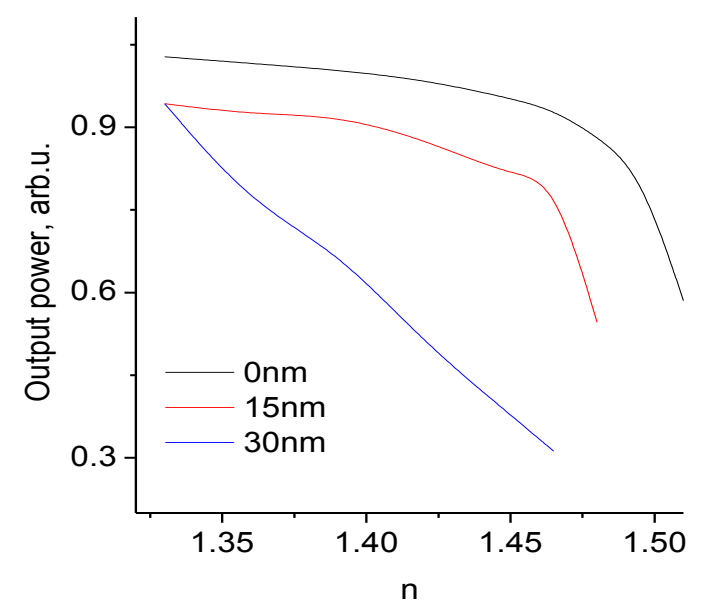

Fig. 6. Output signal power as a function of a cover refractive index for different buffer layer thickness.

In summary, the paper presents the possibility of applying a high-index buffer layer located between the core of a multimode section and the dielectric sensing layer.

The purpose of the buffer layer was to focus the energy field very close to the sensing layer and thereby increase the impact of cladding parameters changes on the output signal. For a thicker buffer layer the modal field moves more toward the cladding layer and, as a result, a greater change of the output signal is obtained.

The work is financed by the Polish National Science Centre "NCN" within the grant 2012/07/B/ST7/01 471.

\section{References}

[1] V. Passaro et al., Sensors. 12, 15558 (2012).

[2] P.V. Lambeck, Measur. Sci. Technol. 17, R93 (2006).

[3] Hua, Ping et al., Sens. Actuat. B-Chem. 87, 250 (2002).

[4] E. Maciak, et al., Molecular and Quantum Acoustics 26, 205 (2005).

[5] G. Stewart, B. Culshaw, Opt. Quant. Electron. 26, 249 (1994).

[6] A. Szewczuk, M. Blahut, Acta Phys. Pol. A 118, 1254 (2010).

[7] A. Szewczuk et al., Acta Phys. Pol. A 118, 1250 (2010).

[8] A. Szewczuk, M. Blahut, Opt. Appl. 43, 441 (2013).

[9] A. Szewczuk, M. Blahut, Acta Phys. Pol. A 124, 570 (2013).

[10] K.R. Kribich et al., Sens. Actuat. B-Chem. 107, 188 (2005).

[11] A. Opilski et al., Opt. Eng. 36, 1625 (1997).

[12] D. Kasprzak, M. Blahut, Proc. of the 14th European Conference on Integrated Optics ECIO 14, 25 (2007).

[13] L.B. Soldano, E. Pennings, J. Lightwave Technol. 13, 615 (1995). 